\title{
Perceptual aftereffects of sustained convergence
}

\author{
SHELDON M. EBENHOLTZ and DAVID M. WOLFSON \\ University of Wisconsin, Madison, Wisconsin 53706
}

\begin{abstract}
Fixating a target for 6 or 8 min was shown to produce distance aftereffects that varied in direction (i.e., over-or underestimation) and magnitude as a linear function of the reciprocal of the observation distance. Changing fixation during the inducing period also was effective. These aftereffects provide a logical alternative to the assumption of recalibration and serve to challenge the claim of visual change as a result of prism adaptation.
\end{abstract}

It has been known for some time that maintaining the eyes in a state of vertical divergence induces a deviation in the fusion-free position of the two eyes that persists for some time after the inducing stimulation is withdrawn (Ellerbrock \& Fry, 1941). Furthermore, to a certain extent, the degree to which the eyes can be made to diverge will vary with the direction in which the eyes were previously maintained. smaller deviations occurring when the eyes successively assume opposite directions (Ellerbrock \& Fry, 1941). A similar aftereffect has been described which occurs when both eyes assume a common direction, e.g. holding the direction of gaze to the left or right (Carr, 1910; Park, 1969). Thus the fusion retlex which is responsible for maintaining the state of vertical divergence is not necessary for the occurrence of the aftereffect. In both cases, conditions are such that some subset of the six extraocular muscles is innervated and muscle tension is maintained for a short time period. The common aftereffect. in which the eyes are pulled in the direction consistent with prior muscle innervation, follows simply from the premise that the muscle innervation pattern continues beyond the point where either the innervation stimulus is removed (e.g., disparate images in the case of the fusion reflex) or where the volitional command to relax is given.

Yet another class of muscle aftereffects exists, initially reported by Kohnstamm (1915), that follow the same rule. but have been applied typically to skeletal muscle and associated appendages. Perhaps the best known of these effects is the demonstration of the arm that rises, in the absence of any conscious direction. as an aftereffect of sustained pressure against a wall. On the basis of parsimony and in the absence of evidence to the contrary, there is justification for the classitication of the three muscle aftereffects described above as members of a common set of phenomena whose determining condition is

The research was supported in part by Grant MH 13006 from NIMH. The aid of Jean Ebenholtz in running Experiments VIII and $\mathrm{IX}$ and in data analysis is gratefully acknowledged. sustained muscle innervation and whose main characteristic is the continued action of the muscle beyond the point of attempted relaxation. Below, the term "Kohnstamm phenomenon" will be used to refer to the entire class of such events, although perhaps "muscle potentiation effects" (Hughes, 1958) would be equally acceptable.

The authors' interest in the Kohnstamm phenomenon was heightened when it occurred to them that it might serve as a logical basis from which to deduce certain aftereffects of prism adaptation, among them aftereffects of adaptation to altered distance cues (Wallach, Frey, \& Bode, 1972). In a series of ingenious experiments, Wallach and his associates had subjects wear spectacles containing wedge prisms and lenses such that light from nearby objects was refracted as though the objects viewed were far off. Another set of spectacles refracted the light from far objects in a manner equivalent to that of nearby objects seen without the special spectacles. After wearing the prism device for a short time (about 30 min), subjects gave evidence of adapting their distance perception so that objects viewed through the spectacles would tend to be seen at veridical distances. The subjects performed as if the oculomotor cues (accommodation and convergence) and the distance they signaled had come to be recalibrated. For example, it was as if, as a result of viewing objects at optical distances closer than actual distance, the oculomotor cues began to signal an increased distance, thereby moving distance perception toward the veridical. The opposite aftereffect, seeing objects at a decreased distance, was obtained as a result of viewing objects through spectacles at farther than actual optical distances, and these outcomes do indeed support a recalibration hypothesis. However, it is also possible to deduce these results from other grounds. For example, if, as a result of maintained convergence on nearby objects, there was a continued (reflexive) innervation of the convergence mechanism, then in order to fixate on any given target the subject would have to overcome this reflexive innervation by some amount of voluntary innervation in the opposite 
direction, i.e., to diverge. ${ }^{1}$ This in turn would lead to the sense of an increase in distance to the fixated target. The opposite aftereffect would follow from exposure to objects seen at far distances. In this case, there would have to be assumed a reflexive tendency for the eyes to continue to diverge which, when fixating a target, would have to be balanced with a voluntary innervation to converge. This would produce the mistaken impression that the fixated target was too close. Thus, by application of the Kohnstamm phenomenon to the extraocular muscles, it is possible to deduce the identical aftereffects that otherwise are attributable to an active visual adaptation process such as that implied by the recalibration notion (Craske \& Crawshaw, 1974; Wallach, Frey, \& Bode. 1972).

In order to support this line of reasoning, it was first necessary to show that the Kohnstamm phenomenon could in fact occur as a result of sustained tension in the lateral and medial recti, respectively, the two sets of muscles that subserve the convergence mechanism (Adler, 1953; Verhoeff, 1947). Second, it would have to be demonstrated that eye muscle aftereffects can occur with a moving eye rather than merely under stable fixation, if the results were to apply to prism adaptation studies. There was reason for expecting positive results here since it had been shown (Matthaei, 1924) that the Kohnstamm phenomenon occurs under both isometric as well as isotonic conditions, although, since the tension in the contracting muscles of the moving eye varies with ocular position, strictly speaking, one does not have in this case an instance of an isotonic contraction.

\section{EXPERIMENTS I-VIII}

\section{Method}

Initial experiments were performed with a mirror stereoscope, as illustrated in Figure 1. Convergence was varied by making slight adjustments in the position of the targets along a path perpend icular to the optical bench. Since the light path varied only slightly, it could be assumed that the size of the retinal images and the accommodation reflex were held constant (the latter for a distance of about $44 \mathrm{~cm}$ ) while the convergence distance was altered. The test procedure required each subject to fixate a luminous form and to indicate its apparent distance by moving his unseen hand in a slide to a point where it was felt to be at the same distance as the visual target. Two distance estimates were given as practice, followed by two more before and again after an exposure period in which subjects simply fixated a luminous target in an otherwise dark room. Starting positions of the slide were counterbalanced at $10 \mathrm{~cm}$ on either side of the correct position. The posttest-pretest differences were taken as a measure of the aftereffect. Data were derived from seven separate experiments, in which the common features were: eight subjects per group (except Experiment VI, where 10 subjects per group were used), an exposure period of $6 \mathrm{~min}^{2}$ the test target placed at a convergence distance of $29.22 \mathrm{~cm}$, and all targets presented on a mirror stereoscope. With two exceptions, the exposure target was a square outline figure, about $2 \mathrm{~cm}$ to a side, with crossing diagonals, whereas the test figure was an outline circle with a diameter of about $2 \mathrm{~cm}$. The exceptions occurred in those groups in

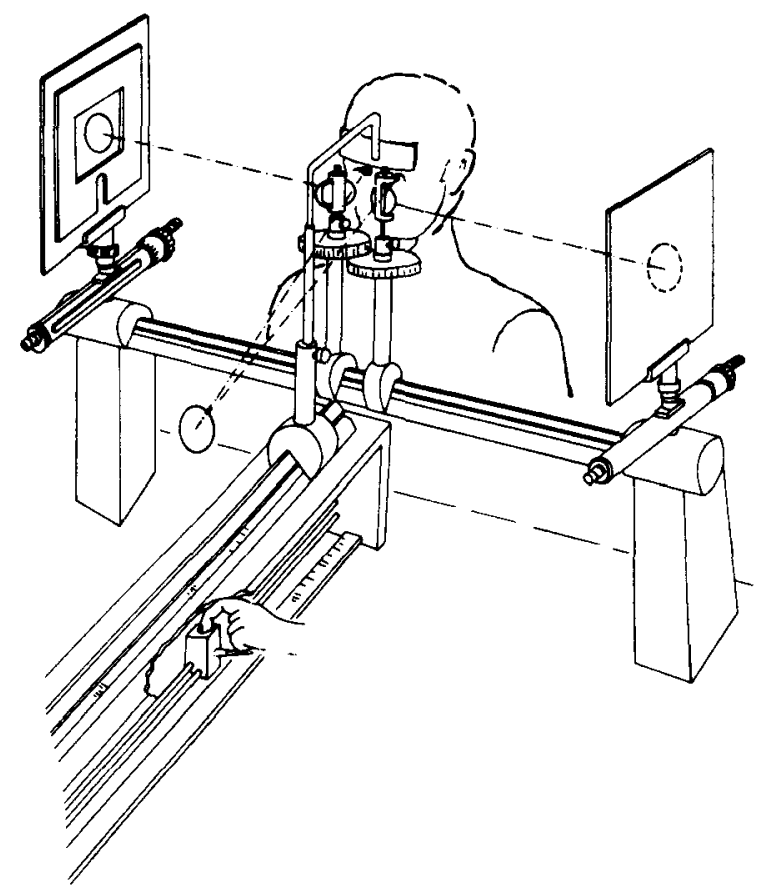

Figure 1. Mirror stereoscope used to present test and exposure targets in Experiments I-VI.

Experiments IV and VI where the test and exposure patterns were reversed (i.e., interchanged), and these are indicated in Figure 2 below by the lowercase " $r$." The unique aspects of the experiments were as follows.

Experiment 1. One group was both exposed and tested at $18.22 \mathrm{~cm}$. a second group at $29.20 \mathrm{~cm}$, and a third group at $40.17 \mathrm{~cm}$. Since the effects of exposure distance and test distance were confounded. further analysis of this experiment was restricted to the data of the second group.

Experiment II. One group was exposed to a target at $16.23 \mathrm{~cm}$ and tested at $29.22 \mathrm{~cm}$.

Experiment III. Same as Experiment II; however. after the initial 6-min exposure period, distance settings were made at 3-min intervals for a total decay period of $9 \mathrm{~min}$, during which the subject sat in the dark with eyes closed.

Experiment IV. Conditions were the same as in Experiments II and 11I, except that exposure and test targets were interchanged and there was no decay interval.

Experiment V. Three groups, run alternately, were exposed to targets at $19.48,22.47$, and $25.63 \mathrm{~cm}$, respectively.

Experiment VI. Two groups, alternately run, each were exposed to targets at $143.08 \mathrm{~cm}$ and tested at $29.22 \mathrm{~cm}$. Test and exposure targets were interchanged for one group relative to the other.

Experiment VI. Two groups, alternately run, were exposed to targets at 22.47 and $58.44 \mathrm{~cm}$, respectively.

\section{Results}

Experiments I and II. In Experiment I, exposure and test at $29.20 \mathrm{~cm}$ yielded overestimation of the target distance for an aftereffect of $1.34 \mathrm{~cm}$. The value was not significantly different from zero, $t(7)=$ $1.30, p>.05$, although six out of eight subjects showed a positive effect. In Experiment II, the exposure target was brought closer to the subject at $16.23 \mathrm{~cm}$, but the test position was the same as in Experiment 1 . This produced a mean level of distance 
overestimation of $4.81 \mathrm{~cm}$, which was significant, t(7) $=4.49, \mathrm{p}<.005$.

Experiments III and IV. A significant aftereffect of $6.22 \mathrm{~cm}$ was produced in Experiment III, $\mathrm{t}(7)=$ $3.95, \mathrm{p}<.005$. Decay means were $3.56,3.76$, and $3.69 \mathrm{~cm}$ after 3,6 , and $9 \mathrm{~min}$, respectively in the dark. Each of the three levels of decay differed significantly (at $p<.05$ ) from the initial level of aftereffect. but they did not differ significantly from each other. It appears as though decay of the distance aftereffect asymptotes at values above the preexposure levels. In Experiment IV, the circular target was used during exposure whereas the square with diagonals served as the test target. A mean level of overestimation of $6.62 \mathrm{~cm}$ was obtained, $\mathrm{t}(7)=7.06$, $\mathrm{p}<.005$. The aftereffect is thus shown not to be contingent on the particular test-exposure target combinations used in Experiments I through III.

Experiment V. The three groups with exposure distances of $19.48,22.47$, and $25.63 \mathrm{~cm}$ differed signiticantly, $F(2,21)=6.03, p<.01$, with means of 4.02. 5.53, and $.86 \mathrm{~cm}$, respectively. All differences involving the latter mean also were significant $(\mathrm{p}<.02)$.

Experiment VI. When the square with diagonals was used as the exposure target at $143.08 \mathrm{~cm}$, the aftereffect produced underestimation, i.e., $-3.10 \mathrm{~cm}$. With the circle as the exposure target, a mean of -6.14 resulted. In each case, the mean was significantly different from zero at $p<.005$. The far observation distance of $143.08 \mathrm{~cm}$ thus clearly produced underestimation, although the two means also differed from each other, $\mathrm{t}(18)=2.49, \mathrm{p}<.05$, with the circle exposure target producing the greater aftereffect. It is not possible, from the present data, to conclude about the cause of this effect, which could reside in the specific pattern, the relative retinal sizes of circle and square, or their relative apparent sizes.

Experiment VII. Exposure at $22.47 \mathrm{~cm}$ produced a mean aftereffect of $3.31 \mathrm{~cm}$, which was significant, $t(7)=2.51, p<.05$. The mean aftereffect of viewing a target at $58.44 \mathrm{~cm}$ was -3.83 and also was significant. $t(7)=2.86, \mathrm{p}<.05$. The two aftereffects differed significantly from each other, $t(14)=3.80$, $\mathrm{p}<.01$, supporting the trend found in the previous experiments for near fixation distances to yield overestimation, with fixation at far targets producing distance underestimation.

In Figure 2, the aftereffects derived from Experiments I-VII were plotted as a function of the reciprocal of the distance to the exposure target. In each case, the test distance was the same at $29.22 \mathrm{~cm}$. The fitted linear function was determined by the method of least squares, average values being used where more than one data point was available at a given exposure-target distance. This yielded a standard error of estimate $\left(\sigma_{\text {est }}\right)$ of .91 , with $r^{2}=$

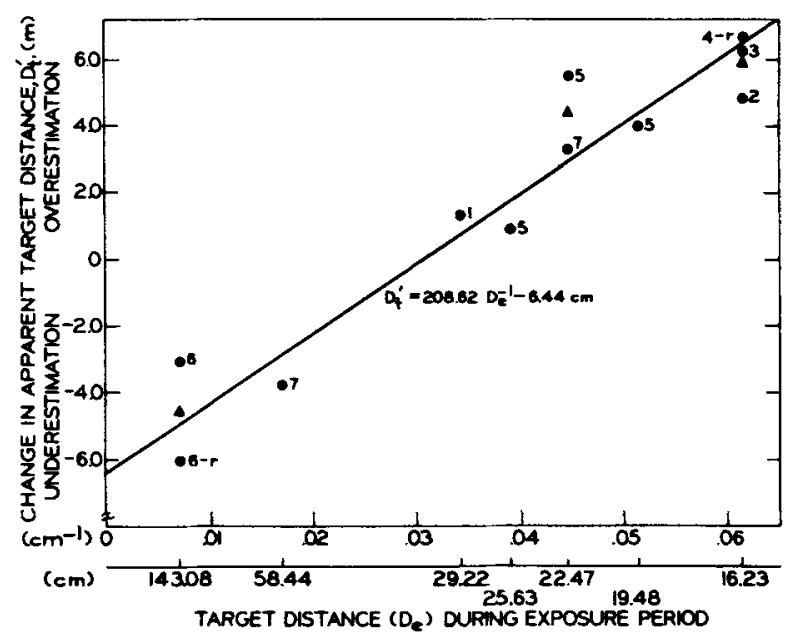

Figure 2. Apparent test-target distance as a function of the reciprocal of the convergence distance during the fixation period. Numerals represent the experiment from which each data point was derived, while the lowercase " $r$ " indicates that test and exposure patterns were interchanged. In all cases, the test pattern was at $29.22 \mathrm{~cm}$.

9497. This indicates that increments in distance to the exposure target account for $95 \%$ of the obtained shifts in apparent distance to the test target. As the exposure distance decreases, the test target appears farther, and with increases in exposure distance, the test target appears closer than it did prior to the 6-min inspection period. This is precisely as required on the assumption that a Kohnstamm-type muscle aftereffect occurs with sustained convergence, the direction of the aftereffect being determined by those muscles (medial or lateral) receiving the greater degree of innvervation. According to the fitted function, fixation at about $32.4 \mathrm{~cm}$ can be expected to yield no aftereffect at all, and this can be interpreted as representing the point of balance in aftereffect between the lateral and medial extraocular muscles of each eye. If the equivalence in aftereffect can be taken as evidence for an equivalence in muscle innervation, then it becomes possible to specify, quite reliably, a physiological position of rest (PPR) for the horizontal vergence system. ${ }^{3}$ Of course, a different position of physiological rest must exist when horizontal version movements are considered.

The $y$-intercept of $-6.4 \mathrm{~cm}$ can be interpreted as the greatest aftereffect possible for sustained far vision (i.e., innvervation of the lateral recti) so long as fixation is limited to a 6-min period under the given conditions of observation. An upper boundary for near fixation (i.e., innvervation of the medial recti) also occurs, since there is a limit to the degree of convergence, i.e., the near point of convergence, and the data suggest that it too is in the region of a $6-\mathrm{cm}$ aftereffect. 


\section{EXPERIMENT VUI}

The second requirement of the research was to justify the application of the Kohnstamm phenomenon to the case in which fixation changed from target to target. This is a necessary step if the Kohnstamm phenomenon is to be relevant to the study of prism adaptation where the subject usually is free to fixate objects over a range of distances.

\section{Method}

Three groups of eight subjects each were exposed to a set of four red-light-emitting diodes. about $.6 \mathrm{~cm}$ in diam, arranged in equal intervals. one behind the other, at eye level in the subject's median plane. For all three groups, the nearest diode was tixed at $15 \mathrm{~cm}$. whereas the fourth diode was set at $27.8,50.0$, or $120.0 \mathrm{~cm}$ from the subject, depending upon the group. This produced ranges of 12.8 . 35.0 , and $105.0 \mathrm{~cm}$ for Groups $\mathrm{N}$ (near). $\mathrm{M}$ (medium), and $\mathrm{F}$ (far), respectively. Pre- and posttests were as previously described. The test target was placed at $35.0 \mathrm{~cm}$ from the subject and had the same pattern as the exposure target of the previous experiments. During an 8-min exposure period, the subject fixated the four diodes, for $5 \mathrm{sec}$ each, in succession, while the remaining lights were out. There was a 1-sec dark interval between fixations. Within each group. two different near-to-far target fixation sequences and two far-to-near sequences were used, with each diode fixated an equal number of times during the exposure period.

\section{Results}

The mean differences between pre- and postexposure distance settings were $3.97,-.19$, and $-2.42 \mathrm{~cm}$ for Groups N, M, and F, respectively, the negative sign indicating underestimation. The data are represented in Figure 3, where the smooth function represents a fit by the method of least squares. The means of Groups $N$ and $F$ were

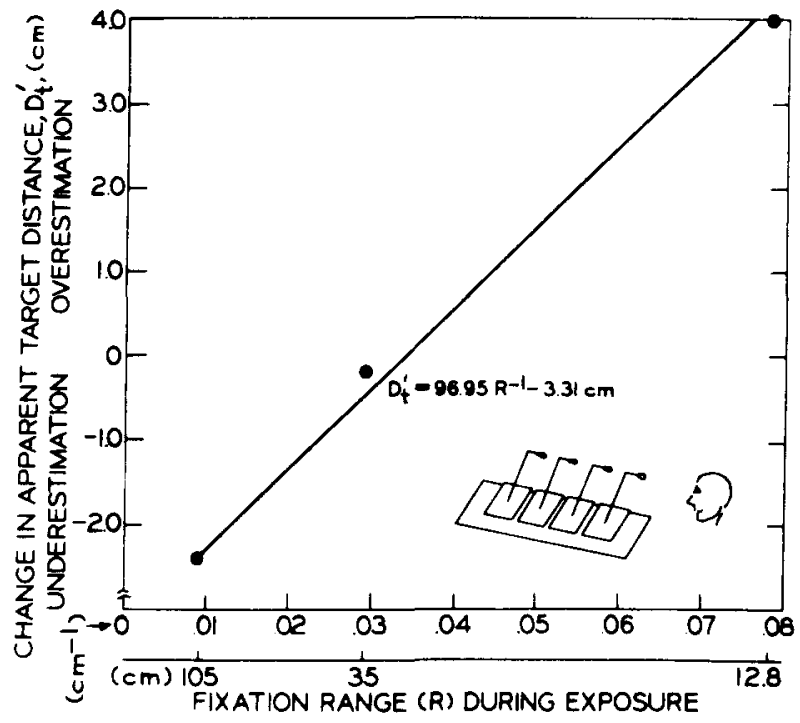

Figure 3. Apparent test-target distance as a function of the reciprocal of the fixation range during exposure. Inset shows the placement of the fixation targets at the near range. significantly different from zero, $t(7)=4.67(\mathrm{p}<.01)$ and $1.91(\mathrm{p}<.05)$, respectively. Group $M$ settings, however, did not differ significantly from zero, $t(7)=$ $-.20, p>.05$. Analysis of variance yielded $F(2,21)=$ $9.64, \mathrm{p}<.005$, indicating that the three ranges produced significantly different aftereffects, viz, overestimation at the near range, no effect at the medium range, and underestimation at the far range.

\section{EXPERIMENT LX}

The purpose of this study was to provide a further examination of the distance aftereffect when fixation varied during the inducing period and also to further probe the relation between degree of convergence and aftereffect magnitude.

\section{Method}

Two groups of 12 subjects each were exposed to a pair of red-light-emitting diodes for a total of $8 \mathrm{~min}$. For Group $\mathrm{N}$, these were placed one behind the other at eye level, at 18.17 and $23.28 \mathrm{~cm}$ from the subject, whereas Group F viewed the targets from 62.08 and $248.30 \mathrm{~cm}$, respectively. The diodes were fixated alternately for 5 sec each with a 1-sec dark interval, so that only one light was visible at any one time. The test target consisted of a circular array of small spots of light surrounding a center spot. The pattern had a diameter of approximately $1.25 \mathrm{~cm}$ and was placed at $33.86 \mathrm{~cm}$ during the test trials. For a hypothetical subject with an interocular axis of $6.5 \mathrm{~cm}$, the convergence angle associated with the test target was $11.0 \mathrm{deg}$. When focusing on the near and far targets in Condition $\mathrm{N}$, convergence angles of 20.5 and $16.0 \mathrm{deg}$, respectively, were formed. For Condition $F$, corresponding values were 6.0 and $1.5 \mathrm{deg}$, respectively. Thus, in each case, the change in convergence from near to far target was $4.5 \mathrm{deg}$, and this interval was symmetrical around the test target convergence angle of $11.0 \mathrm{deg}$. Pre- and posttests were taken as in the previous studies. In addition, after the $8-\mathrm{min}$ induction period, three decay tests were made at 4 -min intervals.

\section{Results}

Means of 1.8 and $-3.5 \mathrm{~cm}$ were achieved by Groups $N$ and $F$, respectively, and both values were significantly different from zero $(p<.05)$. The means also differed significantly from each other, $t(22)=$ 3.93. $p<.005$, showing, as in the previous studies, distance overestimation after fixating near targets, underestimation when fixating over a range of far targets.

The decay tests showed a shift in the aftereffect of both groups toward the preexposure level and, as in Experiment III, a tendency to asymptote short of this value. For Group $\mathrm{N}$, decay means were $0.4,0.4$, and $0.8 \mathrm{~cm}$, after 4,8 , and $12 \mathrm{~min}$, respectively, with eyes closed in the dark. Comparable values for Group $F$ were $-2.1,-1.4$, and $-1.3 \mathrm{~cm}$.

The distance aftereffect is represented in Figure 4 as a function of the reciprocal of the geometric mean distance to the fixation targets. Although only two points were available for this function, it is nevertheless informative on two points. First, the point at which positive and negative aftereffects 
cancel was, by interpolation, $28.6 \mathrm{~cm}$, a value very close to the 29.4-cm value obtained in Experiment VIII. Second, the aftereffects obtained by Groups $\mathrm{N}$ and $\mathrm{F}$ can be shown to be virtually perfectly symmetrical about the value, $-0.8 \mathrm{~cm}$, corresponding to .029 on the abscissa of Figure 4. The latter represents the $11.0-\mathrm{deg}$ convergence angle $(33.86 \mathrm{~cm}$ distance) around which the near and far sets of fixation points also were symmetrically placed. This is precisely what a linear relation between aftereffect magnitude and reciprocal exposure distance (or convergence angle) would require.

\section{DISCUSSION}

The first set of experiments established that sustained convergence over a 6-min exposure period was capable of producing aftereffects in apparent distance. These aftereffects were shown to yield overand underestimation as well as null effects, depending upon the given convergence distance. Since a mirror stereoscope was used both during exposure and test periods, the stimulus to accommodation as well as image size was held constant at all exposure and test distances, respectively. Consequently, these potential distance cues cannot be responsible for the differential distance settings actually obtained. Nor can the relative image sizes of the test and exposure targets have produced these effects, since this aspect of stimulation also was invariant over convergence distance. It is conceivable, however, that the conflict between the two distance cues of accommodation and (fusional) convergence could have served to produce a recalibration of the latter in terms of the former. It follows that since accommodation distance was fixed at about $44 \mathrm{~cm}$, no aftereffect should occur at this convergence distance. Yet the function represented in Figure 2 shows a null point at around $32 \mathrm{~cm}$. Furthermore, the presence of aftereffects in Experiments VIII and IX, where a synchronous relation held between accommodation and convergence, also yields no positive support for the convergence-accommodation conflict hypothesis. The aftereffects would seem to be best understood as instances of the Kohnstamm phenomenon in which muscle innervation outlasts the stimulus to innvervation itself.

Experiments VIII and IX demonstrated the presence of distance aftereffects even where fixation varied over a large range of target distances. Thus, it can be concluded that a constant convergence distance is not required in order to produce the aftereffects, but rather it seems sufficient to maintain tension in the extraocular muscles in a manner analogous to isotonic muscle stimulation. The most notable difference between the static and dynamic conditions of aftereffect production appears to be in the rate at which the aftereffect changes with

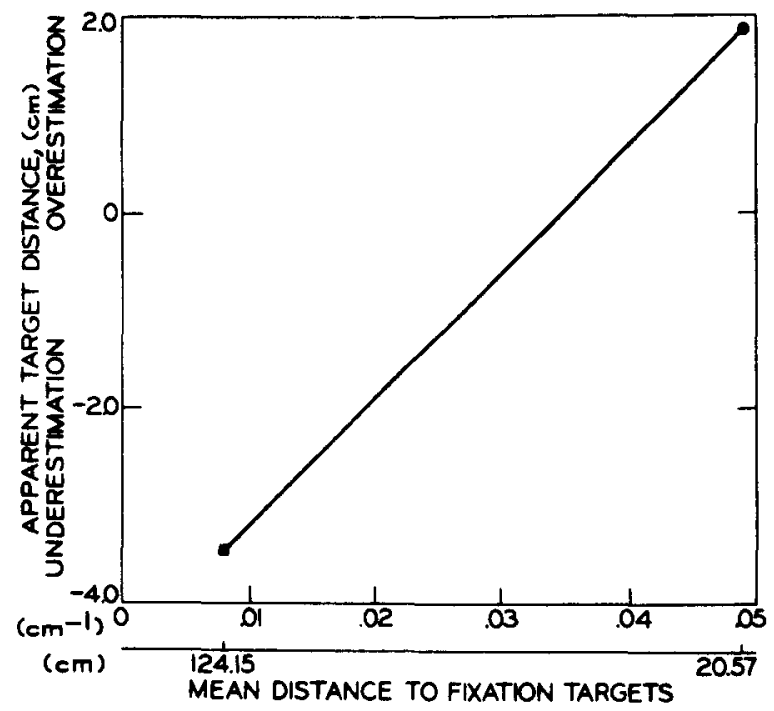

Figure 4. Apparent test-target distance as a function of the reciprocal geometric mean distance to the pair of fixation targets.

increments in fixation distance. A comparison of the slopes represented in Figures 2 and 3 indicates a reduction in slope by more than one-half where fixation was permitted to vary from target to target during the exposure interval. ${ }^{4}$ This outcome probably reflects the fact that during movement there is the relation of reciprocal inhibition among the agonist-antagonist muscle pairs. Thus, while diverging, the medial recti will be inhibited and the lateral recti innvervated, with the opposite pattern holding during convergence. Because of the innvervation of the agonist, during movement, the Kohnstamm phenomenon would tend to be distributed across both the lateral and medial muscles, and hence to balance out. Furthermore, because of the period of inhibition, during movement, the antagonist is provided the opportunity to recover from the tension that would otherwise contribute to the Kohnstamm phenomenon. For both reasons, therefore, aftereffects may be expected to be diminished during movement as compared with static fixation conditions, the former requiring greater exposure periods to reach a given level of aftereffect.

Craske and Crawshaw (1974) obtained similar (near) distance aftereffects resulting from a 3-min inspection period of the feet, but no significant effects when control subjects inspected a disk. The present authors' reanalysis of their data showed no significant differences between experimental and control means and a control mean significantly different from zero, $t(15)=1.91, p<.05$, based upon a one-tailed $t$ test. Craske and Crawshaw based their conclusion, that the control group showed no effect, on a two-tailed F test. In view of the present findings, the marginal effect is to be expected because of the small inspection interval 
and also because the observation distance was near the point at which positive and negative aftereffects balance. Since the subjects wore base-out 10 diopter prisms while viewing a target at $1.5 \mathrm{~m}$, we calculate the convergence distance to be $26.8 \mathrm{~cm}$. A balance point of $29.4 \mathrm{~cm}$ was found in Experiment VIII and $32.4 \mathrm{~cm}$ using the data of Experiments I-VII.

The ranges investigated in Experiment VIII extended from a constant near point of $15.0 \mathrm{~cm}$ to far points of 27.8, 50.0 , and $120.0 \mathrm{~cm}$. The same foreshortened range of convergence could be produced by wearing pairs of base-out wedge prisms with magnitudes of $11.75,6.54$, and 2.72 prism diopters, respectively. Thus, the present research is analogous with studies of adaptation employing base-out prisms. Convergence-cue adaptation to wedge prisms has been investigated (Craske \& Crawshaw, 1974; Wallach, Frey, \& Bode, 1972; Ebenholtz, Note 1), but only distance overestimation has resulted from base-out spectacles, whereas the present results indicate underestimation at the largest range, corresponding to 2.72 prism diopters. Although such low prism powers have not been investigated in studies where the subject locomotes freely, it is nevertheless unlikely that underestimation would show up unless the subject's pattern of fixations was evenly distributed over the range of observation distances in a manner analogous to that of the present study, where four equal intervals were used. Otheruise, fortuitous circumstances or perhaps a tendency to gaze at nearby objects would govern the fixation distances chosen and the associated sign of the aftereffect as well. The point remains, therefore, that it is the convergence angle and the pattern of innvervation of the extraocular muscles that determine the direction of the aftereffects and not the direction of the prism bases as such.

The point at which no aftereffect should occur was estimated at $32.4 \mathrm{~cm}$ in the first set of studies and at $29.4 \mathrm{~cm}$ in Experiment VIII. The latter was based upon only three data points, but was inferred from conditions in which accommodation and convergence were in synchrony. In contrast, while convergence varied in the first set of studies, accommodation distance was fixed at about $44 \mathrm{~cm}$. The conflict between the different accommodative convergence and fusional vergence cues was absent in Experiment VIII, and hence estimates of the points of balance between the medial and lateral muscles, i.e., the position of physiological rest, probably are more reliable in that case.

The distance aftereffects described above were achieved under conditions in which it is highly unlikely that a reclibration could be obtained between a given state of convergence and the apparent distance with which it is normally associated. This is, perhaps, especially clear in Experiments VIII and IX, where only veridical distance cues were present and where, therefore, no cue contlict was possible. The obtained aftereffects, however, are predictable from two assumptions, viz, (1) that the Kohnstamm phenomenon is applicable to the extraocular muscles, and (2) that reflexive, as compared to voluntary. innvervation is either not monitored on the outflow side or does not produce sufficient inflow signals to contribute to proprioception. Thus, for convergence morements, the Kohnstamm phenomenon is equivalent to the presence of a hidden load attached to the medial or lateral extraocular muscles. The load, although not directly sensed, necessitates extra innvervation to be issued in order to attain any given convergence posture. The apparent distance must then vary simply because the magnitude of innvervation also has varied.

Given this account of distance aftereffects, the question must remain open as to whether recalibration does indeed occur at all. The question goes further than adaptation of the convergence cue, since the lateral direction of gaze also is subject to a Kohnstanm aftereffect (e.g.. Park, 1969). This is equally true of the eye-specitic adaptation paradigms (e.g., Ebenholtz, 1970; Foley, 1974; Hajos, 1968), where, as in the present study, because of the fusion reflex, the prisms produce opposite deviations of the eyes during the adaptation period. Because of the Kohnstanin phenomenon, as applied to the extraocular muscles, these induced innervation patterns may be assumed to outlast the period of stimulation and, along with errors in apparent distance, should be manifest either as a deviation of the visual axis of each eye from straight ahead or as an error in the apparent location of a fixated target. Craske and Crawshaw (1974) have provided some positive evidence for these inferences. The present evidence and the analysis offered would suggest that any claims for visual change or recalibration as a result of prism adaptation must be conservatively reevaluated.

\section{REFERENCE NOTE}

1. Ebenholtz, S. M. The possible role of eye muscle potentiation in several forms of prism adaptation. Presented at the Conference on the Recombination Procedure as a Tool for the Study of Visual Perception, Lawrence, Kansas, 1973.

\section{REFERENCES}

Adler, F. H. Pathologic physiology of strabismus. A.M.A. Archives of Ophthalmology, 1953, 50, 19-29.

CARr, H. A. The autokinetic sensation. Psychological Review, $1910,17,42-75$.

Craske, B. \& Crawshaw, M. Adaptive changes of opposite sign in the oculomotor systems of the two eyes. Quarterly Journal of Experimental Psychology, 1974, 26, 106-113.

Ebenholtz, S. M. On the relation between interocular transfer of adaptation and Hering's law of equal innvervation. Psychological Review, 1970, 77, 343-347. 
Ellerbrock, V., \& Fry, G. A. The aftereffect induced by vertical divergence. American Journal of Optometry, 1941, 18. 450-454.

Foley, J. E. Factors governing interocular transfer of prism adaptation. Psychological Review, 1974, 81, 183-186.

Hajos, A. Sensumotorische Koordinationsprozesse bei Richtungslokalisation. Zeitschrift für Experimentelle und Angewandte Psychologie, 1968, 15, 435.461.

Hughes, J. R. Post-tetanic potentiation. Physiological Reviews, 1958, 38, 91-113.

КонNstamm, O. von. Demonstration einer katatonieartigen Erscheinung beim Gesunden (Katatonusversuch). Neurologisches Zentralblatt, 1915, 34, 290-291.

Levy, J. Physiological position of rest and phoria. American Journal of Ophthalmology, 1969, 65, 706-713.

MatthaEI, R. Nachbewegungen beim Menschen. (Untersuchungen uber das sog. Kohnstammsche Phänomen). Pflügers Archiv für die gesamte Physiologie, 1924, 202, 587-600.

PARK, J. N. Displacement of apparent straight ahead as an aftereffect of deviation of the eyes from normal position. Perceptual and Motor Skills, 1969, 28, 591-597.

VerhoefF, F. H. Problems concerning convergence. Transactions of the American Academy of Ophthalmology and Otolaryngology, 1947, 52, 15-19.

Wallach, H., Frey, K. J., \& Bode, K. A. The nature of adaptation in distance perception based on oculomotor cues. Perception \& Psychophysics. 1972, 11, 110-116.

\section{NOTES}

1. "Diverge" refers to vergence movements from near to far points.

2. Pilot studies showed an increase in aftereffect with exposure time. with reliable effects obtained at $6 \mathrm{~min}$ for $n=8$.

3. Levy's (1969) procedure for determining the PPR was based upon the slow drift movements of the eye toward the PPR at which point errors in target position due to eye movements were assumed to be random in direction. The present procedure appears to permit estimates of PPR with relatively low variance. The balance point (i.e.. PPR) obtained in Experiments I-VII was $32.4 \mathrm{~cm}$, corresponding to a convergence angle of $11.7 \mathrm{deg}$, while Experiment VIII yielded a PPR of $29.4 \mathrm{~cm}$ for a convergence angle of $12.7 \mathrm{deg}$. Levy obtained $9.6 \mathrm{deg}$ as the difference in PPR determined for each eye separately.

4. This occurred despite the increased exposure interval from 6 to $8 \mathrm{~min}$ and an increase in test target distance from 29.2 to $35.0 \mathrm{~cm}$. both of which factors may be expected to increase the subject's distance setting.

(Received for publication November 25, 1974; revision received February 6,1975 .) 